\section{Detection and placement of redundant signal elements in tachistoscopic displays of letters*}

\author{
ELIZABETH LIGON BJORK $†$ and WILLIAM K. ESTES \\ The Rockefeller University, New York, N.Y. 10021
}

On each trial of a forced-choice detection experiment, $S$ indicated which of two signal elements was represented and also specified the cell of the 5 by 5 matrix display in which he detected the signal. Reaction time on correct choice, correct placement trials proved virtually invariant with respect to number of redundant signal elements present in the display. These data do not support a serial model for sensory processing, but they could be accommodated by a parallel model for sensory processing together with a serial self-terminating model for processes involving comparison of elements of a display with items in memory. Further analyses of both frequency and reaction time data support the assumption that the distributions of observed detection response measures represent mixtures of true detections, guesses, and misperceptions

One of the principal types of models studied in relation to visual signal detection assumes that the information required to identify one of two alternative signal elements in a tachistoscopic display is extracted by a self-terminating, character-by-character scanning process (Bamber, 1969; Estes \& Taylor, 1964, 1966; Sperling, 1963). If so, and if the scanning is of the order of $10-40 \mathrm{msec}$ per character, as assumed in the formulation of these models to date, then response time should be a decreasing function of the number of redundant signal elements in a display of constant size.

Consider, for example, a 5 by 5 matrix including $\mathrm{k}$ redundant signal elements and $25 \cdot \mathrm{k}$ noise elements (as used in a recent study by Wolford, Wessel, \& Estes, 1968, and also in the experiment to be reported). If there is but one signal element in the display, on the average it will be the 13th character reached in the course of a serial scan; if there are two redundant signal elements, one of them will be reached, on the average, at the $9 \mathrm{th}$ step of a serial scan; and if there are three redundant signals, one will be reached at the 6 th or 7 th step (mean 6.5).

A recent study by Bamber (1969), utilizing a same-difference procedure rather than forced-choice detection, yielded some support for this type of serial model, response time as a function of the number of elements (1-4) differing between two successively presented displays being accurately described by the self-terminating scanning model on the assumption that the rate of processing is approximately $25 \mathrm{msec}$ per character. Contrariwise, a study by Wolford, Wessel,

\footnotetext{
*This research was supported in pare by Public Health Service Grant GM16735 Irom the National Institute of General Medical Sciences.

$\ddagger$ Now at the University of Michigan.
}

and Estes (1968), utilizing a forced-choice detection procedure, yielded no support for the model. Although reaction time on correct detection trials decreased as a function of number of redundant elements, it proved possible to account fully for this trend in terms of a changing mixture of two response time distributions, the one with the lower mean obtaining on true detection trials and the one with the higher mean obtaining on trials when responses arose from guessing. When the raw data were corrected for the changing proportions of guesses as a function of number of redundant signals, either by an algebraic procedure or by utilizing confidence judgments, the curve relating response time to number of redundant signals became horizontal. A related study by Atkinson, Holmgren, and Juola (1969), utilizing a yes-no detection procedure, yielded similarly negative results.

The possibility must be considered that the negative results of the studies by Atkinson, Holmgren, and Juola and Wolford, Wessel, and Estes are attributable to inadequacies in controlling motivation and other aspects of task orientation on the part of the $S$ or by limitations on the analyses of the data. With respect to the former, it seems reasonable to expect that a serial scanning process might be self-terminating only if $\mathrm{Ss}$ are adequately motivated to respond as quickly as possible on each trial. If the scanning process is to some extent under voluntary control, or in any event modifiable by practice, then in the absence of adequate motivation for fast responding, the scanning might continue on each trial until all elements in the display have been inspected rather than terminating upon detection of a signal element

With regard to the second possible source of difficulty, the negative results, especially those of Wolford, Wessel, and
Estes, are subject to the possibility that the procedures used to estimate response times on true detection trials, uncontaminated by responses arising from guessing, were not fully adequate. In the present study, we have attempted to improve on the preceding ones in both of these respects.

The experiment to be reported will be, in essentials, a replication of one of those reported by Wolford, Wessel, and Estes, but with a number of modifications in procedure. The task is forced-choice signal detection from 5 by 5 matrices of noise and signal letters. Each display includes zero, one, two, or three instances of one or the other of two signal letters ( $A$ or $\mathrm{T}$ ), the rest of the cells being filled with randomly drawn "noise" letters. The task of the previous experiments has been modified by requiring the $S$ on each trial not only to indicate which signal element he believes to have been represented in the display, but also to indicate the cell in which it appeared. The assumption is that data for trials on which the $S$ gives a correct detection response and locates the signal element in the proper cell will be relatively free of guesses. Also, several measures involving manipulations of incentives were laken in order to insure that the $S$ respond as quickly as possible on each trial. It was anticipated that analyses of the combined detection and placement data would also yield some new information concerning the sources of errors in this situation.

\section{Apparatus \\ METHOD}

The tachistoscope used in this experiment has been described by Estes and Taylor $(1964,1966)$ and by Wolford, Wessel, and Estes (1968). As in the latter study, a viewing tunnel with an attached goggle frame placed the $S 24$ in. from the stimulus field and assured a standard visual orientation for all Ss. The pre- and postexposure fields were dark except for the presence of a dim fixation point located in the center of the stimulus field which remained on throughout the experiment. The stimulus exposure field had a brightness of $1.3 \mathrm{fc}$. The $\mathrm{S}$ responded by pressing one of two telegraph keys, placed directly beneath the viewing tunnel and in front of the $S$. The keys required $13 \mathrm{~g}$ of force to stop the timer.

\section{Subjects}

Three female Rockefeller University laboratory technicians and one female college student served as $S$ s in the experiment and were paid for their participation at the rate of $\$ 2$ per hour. Each $S$ served in 12 th experimental sessions, with only the data from the last 5 sessions being used in the analysis. 


\section{Stimulus Materials}

The stimulus materials were 5 by 5 arrays of English letters typed on white $8 \times 5$ in. cards with IBM Directory type. Each card contained zero, one, two, or three signal elements, with the rest of the array being filled from the 24 remaining English letters without duplication. The signal elements were either As or Ts. For every stimulus card containing one or more As as the signal elements, there was constructed an identical card except that Ts appeared as the signal elements. The signal elements were placed randomly within the array except for the restriction that no more than two signal elements could appear in adjacent row or column cells. A total of 500 cards was prepared, of which $10 \%$ contained no signal element and $30 \%$ each contained one, two, or three signal elements. From these 500 cards, three samples of 120 cards each were drawn; each sample contained 12 zero-element cards, and 36 each of one-, two-, and three-signal element cards. The S received all the cards in one sample during each experimental session. Which sample the $S$ received in any given session was determined randomly, except for the constraint that each $S$ receive all three samples approximately equally often and never the same sample twice in immediate succession. The 5 by 5 arrays subtended a visual angle of $2 \mathrm{deg}, 30 \mathrm{~min}$.

\section{Procedure}

Each experimental session consisted of 20 practice trials followed by 120 data collection trials. The stimulus cards were presented in a random order. Each trial began with the $E$ informing the $S$ that the stimulus card was in position. As soon thereafter as she felt ready, the $S$ pressed a foot pedal which initiated the presentation of the stimulus card for a duration of $50 \mathrm{msec}$. The $\mathrm{S}$ then responded by pressing either the A or the T key. Following this detection response, the $S$ was required to check a cell position on an unfilled 5 by 5 matrix to indicate in which position the signal element had been located on that trial. The $\mathrm{S}$ was told to respond absolutely as rapidly as possible while still being as accurate as possible. Explicit instructions were given to respond immediately upon detecting a signal element and not to continue searching. If the $S$ thought she detected more than one signal element she could check more than one position in the matrix, but she was to regard such trials as an indication that she was not responding as quickly as possible. In addition, monetary rewards were offered for reducing average response time from one session to the next and for obtaining the greatest number of fast accurate detection responses. The Ss were given unlimited time for both the detection and the placement response. One experimental session required approximately $50 \mathrm{~min}$.

\section{RESULTS}

The results to be reported are based on pooled data for the four Ss over Days 8-12. Uniformity of all principal relationships in the data from $S$ to $S$ was comparable to that observed in previous studies (Estes \& Taylor, 1966; Wolford, Wessel, \& Estes, 1968).

The overall percentages of correct detections were 63,76 , and 81 for displays including one, two, or three redundant signal elements, respectively. These values follow a similar trend but are somewhat lower than those of the corresponding experiment by Wolford, Wessel, and Estes (1968). The basis for the difference evidently could not be degree of practice, since there were no appreciable changes in level of correct responding over the portion of the present study represented in these data.

A complication in the interpretation of the aspects of our data having to do with placement responses arises from the fact that the Ss sometimes marked the locations of two signal elements on a trial. These double placement responses occurred on $2 \%, 7 \%$, and $11 \%$ of the trials for the one, two, and three signal conditions, respectively. Therefore, in computing the percentages of correct detection and placement responses shown in Table 1 , we have utilized only the data from trials on which single placement responses were made.

The proportions of correct detections on trials when correct placement responses occurred, presented in the third row of Table 1, suggest that the detection responses on these trials can be considered to be largely true detections with a relatively small proportion of contamination by guessing responses. Thus we shall assume that it is reasonable to use the data for trials on which both detection and placement responses were correct as a basis for estimating latencies of true detections.

A bit of evidence concerning the nature of placement errors can be obtained from a breakdown of these errors on correct detection trials according to the distance of the placement responses from the cell containing the signal. For the one-signal condition, $26 \%, 20 \%, 21 \%$, and $34 \%$, respectively, of these placements fell at city block distances of $1,2,3$, or 4 or more cells from the signal. If these placement responses were distributed at random, the corresponding expected percentages would be $13,19,23$, and 45 . The deviations from
Table 1 Percentage of Correct Detection and Placement Responses*

Number of Signal Elements

\begin{tabular}{lccc} 
& \multicolumn{3}{c}{ Number of Signal Elements } \\
\cline { 2 - 4 } Type & 1 & 2 & 3 \\
\hline$D$ & 62 & 75 & 79 \\
$P$ & 25 & 44 & 46 \\
$D$ given $P$ & 94 & 97 & 97 \\
$D$ given $\bar{P}$ & 52 & 57 & 63 \\
$P$ given D & 37 & 57 & 56 \\
$P$ given $\bar{D}$ & 4 & 5 & 7 \\
\hline
\end{tabular}

* Data for trials on which single placement responses were made.

${ }^{*} D$ denotes correct detection, $\bar{D}$ incorrect detection, $P$ correct placement, $\vec{P}$ incorrect placement.

chance, though not large, are doubtless significant, for the data of each individual $S$ follow the same pattern.

An unexpected result is seen in Row 5 of Table 1, where the observed values for percentage of correct placement responses on correct detection trials do not increase with number of signal elements as rapidly as would be expected on the basis of the increased opportunity for correct placement responses to occur by chance on trials when the $\mathrm{Ss}$ were guessing. ${ }^{2}$ Evidently retention of the correct placement response for some reason becomes more difficult as the number of signal elements in the display increases.

Mean latencies, based on all trials of Days 8.12, are presented for each of the principal conditions in Table 2. The one value of primary interest not included in the table is the mean value of $708 \mathrm{msec}$ for trials on which no signal element was present in the display. The decreasing trend for mean correct latency as a function of number of signal elements is similar to that observed in the Wolford, Wessel, and Estes study; also, as in that study, incorrect latencies are uniformly higher than correct latencies, but in this study there is slightly more of a downward trend in error latencies as a function of number of signal elements.

The data of major interest for our present purposes are those represented in the third row of Table 2-the mean latencies of detection responses for trials on which both detection and placement responses were correct. The virtual disappearance of any trend as a function of number of signal elements must be taken to be quite negative evidence with regard to the type of serial processing model discussed in the introduction.

\section{DISCUSSION}

Taking the present results together with those of the earlier study by Wolford, Wessel, and Estes (1968), we now find that three independent methods of estimating 
Table 2 Mean Latencies of Detection Responses in Milliseconds

\begin{tabular}{lccc}
\hline & \multicolumn{3}{c}{ Number of Signal Elements } \\
\cline { 2 - 4 } Response Type & 1 & 2 & 3 \\
\hline Correct & 638 & 590 & 573 \\
Incorrect & 682 & 677 & 643 \\
Correct with Cor- & 547 & 544 & 542 \\
rect Placement & & & \\
\hline
\end{tabular}

reaction times on true detection trials agree in revealing no tendency whatsoever for reaction time to vary as a function of number of redundant signal elements in the manner that should be expected on the basis of a self-terminating serial scanning model. One might be tempted simply to reject that type of model at this point, but there would remain the problem of assimilating these results together with those of Bamber (1969), which seem to support the serial scanning conception.

It seems possible to us that we may find a resolution along the following lines. Suppose that we conclude on the basis of the data of Wolford, Wessel, and Estes (1968) and those of the present study that the extraction of information from the characters of the display to the point of identifying features which differentiate different characters is adequately described by a parallel processing model. Two particular models of this type, formulated at slightly different levels of analysis, are that of Wolford, Wessel, and Estes (1968) and that of Rumelhart (1970). However, the process of comparing the information extracted from the set of characters in the display with the representations in memory of the possible signal elements may well conform to a serial scanning model. The number of possible signals to be scanned in memory is independent of the number of redundant signals present in any particular display and, for each possible signal scanned in memory, only a single decision is required as to whether it matches any element processed from the display. Therefore, processing time should be expected to vary with number of alternative possible signals, but not with number of redundant signals in a display, in a forced-choice detection experiment.

The $S$ in a same-different experiment presumably must compare the representations in memory of corresponding elements of the two displays involved in a trial one at a time, deciding at each position whether or not the two corresponding elements are the same or different. On the assumption that the process of comparison and decision conforms to a self-terminating serial model, we can account for the differences between Bamber's study and the other under consideration. According to this conception, it is the comparison of the visual representation of the character with a representation in memory and a decision as to whether a response criterion is satisfied which requires something of the order of 10 to $40 \mathrm{msec}$ per character.

Even if the mean latency of a true detection response is constant at approximately $540 \mathrm{msec}$, as suggested by our data for correct detection, correct placement trials, the systematic decline in marginal mean latency for correct detection responses as a function of number of redundant signals could be accounted for on the basis of a changing mixture of true detections and guesses (Estes \& Wessel, 1966; Yellott, 1967). Estimating the proportions of true detections and guesses for each signal condition by the usual right-minus-wrong procedure, and assuming that the mean latency of a guess is constant at the value of $708 \mathrm{msec}$ determined from our blank-trial data, we can readily show that the decreasing relative frequency of guesses with increasing number of redundant signals suffices to predict nicely the trend in the top row of Table 2. But on this hypothesis, the values in the second row of Table 2 should be constant at approximately $708 \mathrm{msec}$, and we find instead a decreasing function.

An idea which might lead to a resolution of this problem arises from a recent study by Eriksen and Spencer (1969). Though using a somewhat different experimental arrangement, involving sequential exposure of the characters in a display, these investigators obtained some evidence suggesting that incorrect detections are not random guesses arising in the absence of adequate stimulus input. Rather, they propose that misidentifications occur when incomplete processing of the information associated with a displayed character triggers a response appropriate to another character which shares some features with the one displayed.

A straw pointing in the same direction is to be found in the frequency data of our study. Consider, for example, the last row of Table 1, giving the conditional percentages of correct placements on trials when detection responses were incorrect. If all incorrect detection responses were random guesses, $4 \%, 8 \%$, and $12 \%$ correct placements would be expected by chance for the 1,2 , and 3 redundant signal conditions, respectively. To the extent, however, that errors arise from misidentifications of noise letters as signals, these conditional percentages of correct placements will be driven below the chance level, since the noise letter confused with a signal is necessarily in a position not containing a signal.

Thus we are led to consider the hypothesis that detection responses in our experimental situation should be categorized into three, rather than two, mutually exclusive types: true detections, errors arising from misperception of noise characters as signals, and guesses which reflect no information from the display. Under some circumstances, one might wish to include a fourth category-confusion errors arising when the signal present on a trial is mistaken for the signal not present. However, the results regarding correct placements on error trials, together with the high percentages of correct detections given correct placements (Row 3 of Table 1), tend to support our belief that errors of this type are rare under our experimental conditions.

Under this three-category hypothesis, data for error trials represent a mixture of guesses and misperceptions. If we assume that the mean latency of misperceptions is the same as that for true detections, then the changing mixture of low-latency misperceptions and high-latency guesses can account for the observed decrease in mean error latency with increasing number of redundant signals. It should be noted further that the proportion of misperceptions need not be large. An estimate of $9 \%$ misperceptions, constant over signal conditions, yields quite satisfactory predictions of the observed mean latencies $(627,582$, and 569 for Row $1 ; 688,676$, and 649 for Row $2 ; 549$, 543 , and 542 for Row 3 of Table 2; for details, see Bjork \& Estes, 1970). Thus, it is not surprising that models which assume all errors to be guesses often come off quite well.

\section{REFERENCES}

ATKINSON, R. C., HOLMGREN, J. E., \& JUOLA, J. F. Processing time as influenced by the number of elements in a visual display. Perception \& Psychophysics, 1969, 6, 321-326.

BAMBER, D. Reaction times and error rates for "same-different" judgments of multidimensional stimuli. Perception \& Psychophysics, 1969, 6, 169-174.

BJORK, E. L., \& ESTES, W. K. Supplementary note on detection and placement of redundant signal elements. Technical report, Rockefeller University, 1970

ERIKSEN, C. W., \& SPENCER, T. Rate of information processing in visual perception: Some results and methodological considerations. Journal of Experimental Psychology Monograph, 1969, 79, No. 2.

ESTES, W. K.. \& TAYLOR, H. A. A detection method and probabilistic models for assessing information processing from brief visual displays. Proceedings of the National Academy of Science, 1964, 52, No. 2, 446-454.

ESTES, W. K, \& TAYLOR, H. A. Visual detection in relation to display size and redundancy of critical elements. Perception \& Psychophysics, 1966, 1, 9-16.

ESTES, W. K. \& WESSEL, D. L. Reaction time in relation to display size and correctness of 
response in forced-choice visual signal detection. Perception \& Psychophysics, 1966, 1, 369-373.

RUMELHART, D. E. A multicomponent theory of the perception of briefly presented displays. Journal of Mathematical Psychology, 1970, 7, 191-218.

SPERLING, G. A model for visual memory tasks. Human Factors, 1963, 5, 19-31.

WOLFORD, G. L., WESSEL, D. L., \& ESTES, W. $K$. Further evidence concerning scanning and sampling assumptions of visual detection models. Perception \& Psychophysics, 1968, 3, 439-444.

YELLOTT, J. Correction for guessing in choice reaction time. Psychonomic Science, 1967, 8, 321-322.

\section{NOTES}

1. Data for "same" trials required some augmentation of the model, latencies being shorter than those for "different" trials and varying little with display size.

2. If the $S$ always gives correct placement responses on true detection trials, and otherwise guesses positions at random, then estimates of true detection probabilities from the first row of Table 1 yield predicted values of $.41, .69$, and .77 for the fifth row, grossly overshooting the observed values for two and three redundant signals.

(Accepted for publication September 1, 1970.) 